\title{
Generalized Eigenstate Thermalization Hypothesis in 2D Conformal Field Theories
}

\author{
Anatoly Dymarsky $\oplus^{1,2}$ and Kirill Pavlenko ${ }^{2,3}$ \\ ${ }^{1}$ Department of Physics and Astronomy, University of Kentucky, Lexington, Kentucky 40506, USA \\ ${ }^{2}$ Skolkovo Institute of Science and Technology, Skolkovo Innovation Center, Moscow 143026, Russia \\ ${ }^{3}$ Moscow Institute of Physics and Technology, Dolgoprudny 141700, Russia
}

(Received 24 March 2019; published 13 September 2019)

\begin{abstract}
Infinite-dimensional conformal symmetry in two dimensions leads to integrability of 2D conformal field theories (CFTs) by giving rise to an infinite tower of local conserved quantum Korteweg-de Vries (qKdV) charges in involution. We discuss how the presence of conserved charges constrains equilibration in 2D CFTs. We propose that in the thermodynamic limit large central charge 2D CFTs satisfy generalized eigenstate thermalization, with the values of $\mathrm{qKdV}$ charges forming a complete set of thermodynamically relevant quantities, which unambiguously determine expectation values of all local observables from the vacuum family. Equivalence of ensembles further provides that local properties of an eigenstate can be described by the generalized Gibbs ensemble that includes only qKdV charges. In the case of a general initial state, upon equilibration, the emerging generalized Gibbs ensemble will necessarily include negative chemical potentials and holographically will be described by a quasiclassical black hole with quantum soft hair.
\end{abstract}

DOI: 10.1103/PhysRevLett.123.111602

The topic of thermalization-and, more generally, equilibration of isolated many-body quantum systemshas been an active area of research during the past decade. In the case of nonintegrable systems, i.e., those without an extensive number of local conserved quantities, the emergence of thermal equilibrium has been traced to eigenstate thermalization hypothesis (ETH), which postulates thermal properties of individual energy eigenstates [1-3]. In the simplest form it requires the expectation value of some appropriate (often taken to be local) observable $\mathcal{O}$ in a manybody eigenstate $\left|E_{i}\right\rangle$ to be a smooth function of energy,

$$
\left\langle E_{i}|\mathcal{O}| E_{i}\right\rangle=f_{\mathcal{O}}\left(E_{i}\right)
$$

Qualitatively, Eq. (1) postulates that energy is the only thermodynamically relevant quantity, which completely specifies the local properties of an eigenstate. Condition (1) may apply to all or most eigenstates, in which case it is referred to as strong or weak ETH. The eigenstate thermalization ensures equivalence between the expectation value in the eigenensemble, $f_{\mathcal{O}}\left(E_{i}\right)$, and the thermal expectation value of $\mathcal{O}$ in the Gibbs ensemble, $f_{\mathcal{O}}\left(E_{i}\right)=\operatorname{Tr}\left(e^{-\beta H} \mathcal{O}\right) / Z$, where the effective temperature $\beta$ is fixed through the energy balance relation, $E_{i}=\operatorname{Tr}\left(e^{-\beta H} \mathcal{O}\right) / Z[4]$.

Published by the American Physical Society under the terms of the Creative Commons Attribution 4.0 International license. Further distribution of this work must maintain attribution to the author(s) and the published article's title, journal citation, and DOI. Funded by SCOAP ${ }^{3}$.
When the system is integrable, with an extensive number of conserved charges $Q_{i}$, ETH does not apply. Accordingly emerging equilibrium can be different from the Gibbs state. In this case the equilibrium can be described by the generalized Gibbs ensemble (GGE), a generalization of the grand canonical ensemble that includes an infinite tower of conserved charges [5]. Validity of the GGE has been related to generalized eigenstate thermalization [6-8], which generalizes (1) to include an infinite number of conserved quantities,

$$
\left\langle E_{i}|\mathcal{O}| E_{i}\right\rangle=f_{\mathcal{O}}\left(Q_{k}\left(E_{i}\right)\right) .
$$

Here $\left|E_{i}\right\rangle$ is a mutual eigenstate of the Hamiltonian, charges $Q_{k}, Q_{k}\left(E_{i}\right)$ are the eigenvalues of $Q_{k}$ associated with $\left|E_{i}\right\rangle$, and the function $f_{\mathcal{O}}$ is assumed to be a smooth function of all of its arguments. As in Eq. (1), at the qualitative level, Eq. (2) postulates that charges $Q_{k}$ form a complete set of thermodynamically relevant quantities which fully specify local properties of an eigenstate. Provided that Eq. (2) applies to most states, it ensures equivalence between the generalized microcanonical ensemble and the GGE, establishing validity for the latter to describe the emerging equilibrium, e.g., following a quantum quench [6].

In this Letter we discuss thermalization of twodimensional conformal field theories (CFTs), a rich topic with multiple connections ranging from the cold atom experiments [9] to the physics of quantum gravity [10]. It has been shown that following a quantum quench $2 \mathrm{D}$ conformal theories equilibrate and reach a steady state, which in many cases can be described in terms of the Gibbs 
ensemble [11,12]. At the same time emergence of thermal equilibrium is not universal. Previous studies have shown that the values of different local quantities measured in the individual energy eigenstates differ from their thermal counterparts, unless the central charge is taken to be infinite [13-21]. Furthermore, conformal symmetry in two dimensions gives rise to an infinite tower of local mutually commuting conserved quantum Korteweg-de Vries $(\mathrm{qKdV})$ charges $Q_{2 k-1}$, with the CFT Hamiltonian for the left movers being $Q_{1} \equiv H$, a part of the integrable structure of the 2D CFTs [22-24]. The question we are concerned with is how the presence of these charges affects equilibration. By analogy with the integrable lattice models, it is natural to expect that locally equilibrium states can be described in terms of the GGE, which includes all local $\mathrm{qKdV}$ charges. Indeed, emergence of exactly such a qKdV GGE was analytically shown for a special family of socalled Cardy-Calabrese initial states $[25,26]$.

In the context of integrable systems, the question of which quantities should be included in the GGE is far from trivial. Early studies in the context of $X X Z$ and Lieb-Liniger models have shown that a full set of extensive local charges does not specify local properties of eigenstates, signaling failure of the generalized ETH $[27,28]$. These works raised an important question of the validity of the GGE to describe an emerging equilibrium following a quantum quench [29-31]. A resolution comes from the fact that, besides local conserved quantities, these models give rise to quasilocal conserved charges [32]. Taking them into account restores validity of the GGE [33]. Following studies in the context of integrable field theoretic models, both free and interacting ones, have decisively established that adding quasilocal charges is necessary to accurately describe the after-quench equilibrium state [34-39]. These findings raise an important question emphasized in Ref. [26] if the set of local qKdV charges is generally sufficient to describe equilibrium in large $c$ 2D CFTs, or it should be extended by nonlocal or perhaps some new local charges [40]. In this Letter we show that, at large $c, 2 \mathrm{D}$ CFTs satisfy generalized eigenstate thermalization (2) with the local qKdV charges forming a complete set and ambiguously specifying local properties of the eigenstates.

Our consideration below applies to all large central charge theories with $c \gg 1$. In the case in which theory admits a gravity dual, many aspects can be interpreted holographically. Below we provide holographic interpretation when available without requiring the holographic description to exist.

Two-dimensional conformal field theories admit a split into noninteracting sectors of left and right movers. For simplicity we discuss only one sector explicitly, while all results automatically extend to the full theory. We consider 2D CFT on a circle of the circumference $\ell$ in a mutual eigenstate of all charges $Q_{2 k-1}$,

$$
|E\rangle=\left|\left\{m_{i}\right\}, \Delta\right\rangle, \quad E=Q_{1}=\left(\Delta+\sum m_{i}\right) / \ell,
$$

labeled by the primary state $\Delta$ and the set of integers $\left\{m_{i}\right\}$ [41]. The set $\left\{m_{i}\right\}$ is convenient to parametrize using free boson representation where an integer $n_{k}$ for $k=1,2, \ldots$ specifies the number of times integer $k$ appears in the set $\left\{m_{i}\right\}$. In the thermodynamic limit $\ell \rightarrow \infty$ "energy" $Q_{1}$ and all other qKdV charges are assumed to scale with the system size to yield finite charge densities $q_{2 r-1}=$ $Q_{2 r-1} / \ell$. In terms of $\Delta, n_{k}$ this implies the scaling

$$
\Delta \sim \ell^{2}, \quad \sum_{k} n_{k} k^{2 r-1} \sim \ell^{2 r} .
$$

In what follows we restrict the discussion to the eigenstates (3) with the density charges $q_{2 r-1}=\left\langle E\left|Q_{2 r-1}\right| E\right\rangle / \ell$ which additionally satisfy

$$
\frac{q_{2 r-1}}{q_{1}^{r}}=1+O(1 / c) .
$$

Here and below the CFT central charge $c$ is assumed to be large. Holographically, this regime corresponds to a quasiclassical black hole in three-dimensional anti-de Sitter space $\left(\mathrm{AdS}_{3}\right)$, where 1 on the rhs of Eq. (5) corresponds to classical gravity, while the $O(1 / c)$ term is due to quantum corrections [41-44]. In terms of $\Delta, n_{k}$ an exponential majority of states in the generalized microcanonical ensemble specified by $q_{2 k-1}$ subject to Eq. (5) will satisfy

$$
\frac{\sum_{k} n_{k} k^{2 r-1}}{\Delta^{r}}=O\left(1 / c^{r}\right) .
$$

The typicality of Eq. (6) is justified in the Supplemental Material [45]. In fact Eq. (6) may apply to all states in the generalized microcanonical ensemble (5), yielding a strong version of the generalized ETH in 2D CFTs. To verify that one would need to know the full spectrum of $\mathrm{qKdV}$ charges, going beyond currently known leading $1 / c$ expansion.

In the regime of "quasiclassical gravity" (6), $c \gg 1$, expectation values of $\mathrm{qKdV}$ charges can be calculated explicitly [46],

$$
\begin{gathered}
\ell^{2} q_{1}=\Delta+\sum_{k} n_{k} k, \\
\ell^{4} q_{3}=\Delta^{2}+\sum_{k} n_{k}\left(6 \Delta k+\frac{c k^{3}}{6}\right)+O\left(c^{0}\right), \\
\ldots \\
\ell^{2 r} q_{2 r-1}=\Delta^{r}+\sum_{k} n_{k} p_{2 r-1}(c, \Delta, k)+O\left(c^{r-2}\right),
\end{gathered}
$$


where $p_{2 r-1}(c, \Delta, k)$ are some known polynomials of degree $2 r-1$ which include only odd powers of $k$.

Because of translational invariance the expectation value of a full derivative $\mathcal{O}=\partial \mathcal{O}^{\prime}$ in the energy eigenstate will vanish. Hence it suffices to consider expectation values $\langle E|\mathcal{O}| E\rangle$ only when $\mathcal{O}$ is a quasiprimary operator. Below we consider the case in which $\mathcal{O}$ belongs to the vacuum family, i.e., it is a Virasoro descendant of the identity. To streamline the notations, we introduce $\langle\mathcal{O}\rangle \equiv\langle E|\mathcal{O}| E\rangle$. It is convenient to parametrize $\mathcal{O}$ by its dimension (level). On levels 2 and 4 there are unique quasiprimary operators in the vacuum family,

$$
\mathcal{O}_{2}=T, \quad \mathcal{O}_{4}=T^{2}-\frac{3}{10} \partial^{2} T .
$$

Thus expectation values of $\mathcal{O}_{2,4}$ are identically equal to charge densities $q_{1}, q_{3}$ [47]. On level 6 there are two quasiprimary operators (we always choose quasiprimaries in the basis which diagonalizes Zamolodchikov metric),

$$
\begin{gathered}
\mathcal{O}_{6}^{(1)}=T^{3}-\frac{9}{10}\left(T \partial^{2} T\right)+\frac{4}{35} \partial^{4} T+\frac{93}{70 c+29} \mathcal{O}_{6}^{(2)}, \\
\mathcal{O}_{6}^{(2)}=(\partial T \partial T)-\frac{4}{5}\left(T \partial^{2} T\right)+\frac{23}{210} \partial^{4} T .
\end{gathered}
$$

The expectation value of the combination $\mathcal{O}_{6}^{(1)}+$ $\frac{5}{9}(c / 12) \mathcal{O}_{6}^{(2)}$ is identically equal to $q_{5}$. As in Eqs. (7)(9), at leading order the expectation value of $\mathcal{O}_{6}^{(2)}$ has the form of a polynomial in $\Delta$ and odd powers of $k$,

$$
\left\langle\mathcal{O}_{6}^{(2)}\right\rangle=\frac{9}{5} \sum_{k} n_{k}\left(\frac{c}{6} k^{5}+4 \Delta k^{3}\right)+O\left(c^{0}\right) .
$$

It is possible to use Eqs. (7)-(9) to express any term of the form $\sum_{k} n_{k} k^{2 r-1}$ via $q_{2 j-1}, j \leq r$, but a priori the result would also depend on $\Delta$. Thus, at leading order in $1 / c$, expectation values of $\mathcal{O}_{6}^{(i)}$ are some functions of $\Delta$ and $q_{2 r-1}$. Remarkably, because of the nontrivial cancellations, the final result is $\Delta$ independent and can be expressed solely in terms of $q_{2 r-1}$. To simplify the answer, we introduce dimensionless ratio $q_{2 k-1}=q_{2 k-1} / q_{1}^{k}$ such that $\delta q_{2 k-1} \equiv q_{2 k-1}-1$ is of order $1 / c$. Then $\mathcal{O}_{6}^{(i)}$ measured in units of energy density $q_{1}$ is given by

$$
\begin{gathered}
q_{1}^{-3}\left\langle\mathcal{O}_{6}^{(1)}\right\rangle=1+3 \delta q_{3}+O\left(1 / c^{2}\right), \\
q_{1}^{-3}\left\langle\mathcal{O}_{6}^{(2)}\right\rangle=\frac{9}{5}(12 / c)\left(\delta q_{5}-3 \delta q_{3}\right)+O\left(1 / c^{3}\right) .
\end{gathered}
$$

As we see, different quasiprimary operators have different scalings with $c$. Our calculation applies to leading $1 / c$ behavior of each quasiprimary operator, except for a special one which includes maximal power of $T$ without derivatives. The expectation value of that quasiprimary operator starts with $O\left(c^{0}\right)$, and our result applies to the first two terms in $1 / c$ expansion.

The possibility of expressing eigenstate expectation value $\langle\mathcal{O}\rangle$ as a polynomial in $q_{2 j-1}$ extends to all higher levels. For an operator of dimension $2 r$ the answer depends only on $q_{2 j-1}$ for $j \leq r$. We write down explicit expressions for all operators up to level 10 in terms of $q_{2 j-1}$ in the Supplemental Material [45]. Our results establish generalized eigenstate thermalization for vacuum block observables in large $c$ CFTs.

That expectation value $\langle\mathcal{O}\rangle$ of an operator of dimension $2 r$ includes only qKdV charges $q_{2 j-1}$ up to the same dimension $j \leq r$ can be interpreted as a manifestation of locality. It is analogous to the observation in the context of integrable lattice models that, to describe equilibrium state locally at length scales not exceeding some distance $a$, it is necessary to include only local and quasilocal charges in the GGE with the support within $a$ [48,49].

Our results capture only leading order in $1 / c$. It remains an open question to find exact expressions for the eigenstate expectation values in terms of the $\mathrm{qKdV}$ charges, as was recently done for a particular family of integrable spin models [50,51].

Generalized eigenstate thermalization implies the validity of the qKdV ceneralized Gibbs ensemble

$$
\rho=\exp \left\{-\sum_{k} \mu_{2 k-1} Q_{2 k-1}\right\} / Z, \quad \mu_{1} \equiv \beta,
$$

to describe the local properties of individual energy eigenstates provided that chemical potentials $\mu_{2 k-1}$ are tuned to match values of the eigenstate charges

$$
\ell q_{2 k-1}=\left\langle E_{i}\left|Q_{2 k-1}\right| E_{i}\right\rangle=\operatorname{Tr}\left(\rho Q_{2 k-1}\right) .
$$

Provided that $q_{2 k-1}$, chosen to represent the charge densities of some nonequilibrium initial state $|\Psi\rangle$, a standard argument would consequently equate the GGE expectation values of local operators with those in the diagonal ensemble of $|\Psi\rangle$, written in eigenbasis (3). In most cases the latter would be equal to the expectation values in state $|\Psi\rangle$ upon equilibration. It should be noted though that left and right Hamiltonians $Q_{1}, \bar{Q}_{1}$ are highly degenerate, and therefore the validity of the diagonal ensemble to describe local physics upon equilibration may be violated.

It remains an open question to establish the existence of $\mu_{2 k-1}$, which would solve Eq. (17) for any given set of $q_{2 k-1}$. Using an explicit form of the generalized partition function in the large $c$ limit [46], we can find, up to the $O\left(1 / c^{2}\right)$ corrections, 


$$
\begin{aligned}
\delta q_{2 k-1} & =\frac{q_{2 k-1}}{q_{1}^{k}}-1 \\
& =\frac{24 k}{c} \int_{0}^{\infty} \frac{d \kappa \kappa\left[(2 k-1)_{2} F_{1}\left(1,1-k, 3 / 2,-\kappa^{2}\right)-1\right]}{e^{2 \pi k \gamma}-1}, \\
\gamma & =\sum_{j=1}^{\infty} \tilde{\mu}_{2 j-1} j(2 j-1) \sigma^{j-1 / 2}{ }_{2} F_{1}\left(1,1-j, 3 / 2,-\kappa^{2}\right),
\end{aligned}
$$

where $\tilde{\mu}_{2 k-1}=(\sqrt{6} / \pi) c^{k-1} \mu_{2 k-1}$, and where $\sigma\left(\tilde{\mu}_{2 k-1}\right)$ is positive and satisfies

$$
\sum_{k=1} k \tilde{\mu}_{2 k-1} \sigma^{k-1 / 2}=1
$$

From here it follows that, when all chemical potentials are positive, $q_{2 k-1}$ satisfies an infinite series of inequalities (see the Supplemental Material [45])

$\frac{q_{3}}{q_{1}^{2}}-1 \leq \frac{22}{5 c}+O\left(1 / c^{2}\right), \quad \frac{q_{5}}{q_{1}^{3}}-1 \leq \frac{302}{21 c}+O\left(1 / c^{2}\right)$,

Thus the GGE emerging after equilibration of some general initial state will have to include negative chemical potentials, unless all inequalities (20) are satisfied.

To match the GGE to a primary state all $\mathrm{qKdV}$ densities should be related to each other via $q_{2 k-1}=q_{1}^{k}$ [47]. This is possible only if the integral in Eq. (18) vanishes, which requires $\gamma$ to be infinite. This is consistent with the observation of Ref. [41] that an ensemble with any finite number of nonzero $\mu_{2 k-1}$ cannot describe primary states. This is because in full generality $q_{2 k-1} \geq q_{1}^{k}$, and hence primary states are at the boundary of the phase space of $q_{2 k-1}$ 's. It is nevertheless possible to describe them in the limit, via a GGE with at least some coefficients approaching infinity. The simplest scenario is to consider $\mu_{3}>0$ and arbitrary $\beta \equiv \mu_{1}$, while all other chemical potentials are identically zero. Then in the limit $\tau=\beta\left(6 / \pi^{2} c \mu_{3}\right)^{1 / 3} \rightarrow$ $-\infty$, for all $k, q_{2 k-1} / q_{1}^{k}-1$ will vanishes as $\sim|\tau|^{-3}$, as is shown for $k=2,3$ in Fig. 1.

With just two chemical potentials $\beta, \mu_{3}$ being nonzero, the values of $q_{2 k-1} / q_{1}^{k}-1$ is confined to be between zero and their thermal (Gibbs ensemble) values. This constraint is removed already after turning on one additional chemical potential. For example by taking $\beta, \mu_{5}>0$ and $\mu_{3}<0$, one can fine-tune function $\gamma$ to become arbitrarily small for some positive value of $\kappa$, leading to the divergence of the integral in Eq. (18) and violating quasiclassical regime (5).

From the holographic point of view equilibration in field theory is associated with the formation of a black hole in $\mathrm{AdS}_{3}$, a background dual to the GGE (16). Conserved qKdV charges correspond to the black hole soft hair, which are visible only at the quantum level. At the level of

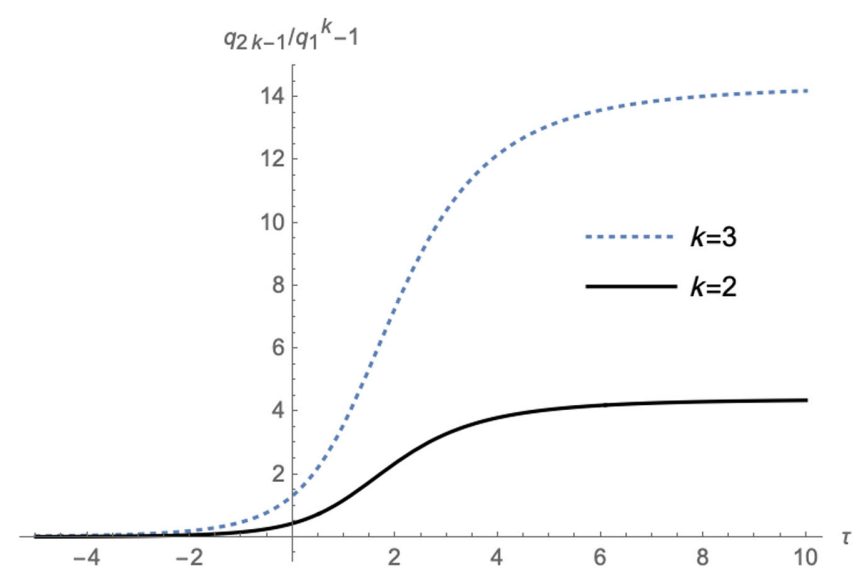

FIG. 1. Plot of $q_{2 k-1} / q_{1}^{k}-1$ in the units of $1 / c$ as a function of $\tau=\beta\left(\pi^{2} /\left(6 c \mu_{3}\right)\right)^{1 / 3}$ for $k=2$, 3. It approaches zero as $|\tau|^{-3}$ for all $k$ when $\tau \rightarrow-\infty$. The opposite limit $\tau \rightarrow \infty$ corresponds to the Gibbs ensemble, $q_{1} \sim \beta^{-1}, \mu_{3} \rightarrow 0$, and $c\left(q_{2 k-1} / q_{1}^{k}-1\right)$, for $k=2,3$ approaching $22 / 5$ and $302 / 21$, correspondingly.

classical gravity $c \rightarrow \infty$, all $\mathrm{qKdV}$ charges are related, $q_{2 k-1}=q_{1}^{k}$. Accordingly there is a unique classical Bañados-Teitelboim-Zanelli black hole family of solutions parametrized by $q_{1}, \bar{q}_{1}[42,43]$ It is an important question to understand the regime $q_{2 k-1} \neq q_{1}^{k}$ holographically, by taking quantum gravity corrections into consideration. This, in particular, should provide holographic interpretation to negative temperature and other chemical potentials, which will necessarily appear starting from a general initial state.

In this Letter we have considered only local probes $\mathcal{O}$ from the vacuum block. In case $\mathcal{O}$ is a nontrivial Virasoro primary, or its descendant, it will have zero expectation value in the GGE (16) for any values of $\mu_{2 k-1}$. This is because in the thermodynamic limit $\ell \rightarrow \infty$ geometry degenerates into a cylinder which is conformally flat. Thus, to satisfy any version of eigenstate thermalization, the eigenstate expectation value $\langle E|\mathcal{O}| E\rangle$ must simply vanish. In terms of the CFT data, this means most or all heavy-heavy-light operator product expansion coefficients must approach zero when the dimension of heavy operators grows to infinity. If that is the case, generalized eigenstate thermalization will be trivially satisfied. It remains an outstanding problem to establish whether large central charge chaotic CFTs, in particular those with gravity duals, exhibit this behavior.

In this Letter we have established that large central charge 2D CFTs in the thermodynamic limit satisfy generalized eigenstate thermalization with the tower of local qKdV charges forming a complete set of thermodynamically relevant quantities. Our analysis has established the universal validity of a generalized Gibbs ensemble that includes all $\mathrm{qKdV}$ charges to describe individual energy eigenstates, and hence in most cases, asymptotic equilibrium states in such theories. It would be important to extend 
the analysis to the next order in $1 / c$, which would reveal whether the eigenstate thermalization is strong-i.e., it applies to all finite energy density eigenstates-or weaki.e., it applies to most such states.

We thank Alex Avdoshkin, Dmitry Abanin, Tomaz Prosen, Marcos Rigol, and Alexander Zhiboedov for the discussions. This work was supported by a grant of the Russian Science Foundation (Project No. 17-12-01587).

[1] J. M. Deutsch, Quantum statistical mechanics in a closed system, Phys. Rev. A 43, 2046 (1991).

[2] M. Srednicki, Chaos and quantum thermalization, Phys. Rev. E 50, 888 (1994).

[3] M. Rigol, V. Dunjko, and M. Olshanii, Thermalization and its mechanism for generic isolated quantum systems, Nature (London) 452, 854 (2008).

[4] L. D'Alessio, Y. Kafri, A. Polkovnikov, and M. Rigol, From quantum chaos and eigenstate thermalization to statistical mechanics and thermodynamics, Adv. Phys. 65, 239 (2016).

[5] M. Rigol, V. Dunjko, V. Yurovsky, and M. Olshanii, Relaxation in a Completely Integrable Many-Body Quantum System: An Ab Initio Study of the Dynamics of the Highly Excited States of 1D Lattice Hard-Core Bosons, Phys. Rev. Lett. 98, 050405 (2007).

[6] A. C. Cassidy, C. W. Clark, and M. Rigol, Generalized Thermalization in an Integrable Lattice System, Phys. Rev. Lett. 106, 140405 (2011).

[7] K. He, L. F. Santos, T. M. Wright, and M. Rigol, Singleparticle and many-body analyses of a quasiperiodic integrable system after a quench, Phys. Rev. A 87, 063637 (2013).

[8] L. Vidmar and M. Rigol, Generalized gibbs ensemble in integrable lattice models, J. Stat. Mech. (2016) 064007.

[9] P. Calabrese and J. Cardy, Quantum quenches in $1+1$ dimensional conformal field theories, J. Stat. Mech. (2016) 064003.

[10] T. Anous, T. Hartman, A. Rovai, and J. Sonner, Black hole collapse in the 1/c expansion, J. High Energy Phys. 07 (2016) 123.

[11] P. Calabrese and J. Cardy, Time Dependence of Correlation Functions Following a Quantum Quench, Phys. Rev. Lett. 96, 136801 (2006).

[12] P. Calabrese and J. Cardy, Quantum quenches in extended systems, J. Stat. Mech. (2007) P06008.

[13] A. L. Fitzpatrick and J. Kaplan, Conformal blocks beyond the semi-classical limit, J. High Energy Phys. 05 (2016) 075 .

[14] F.-L. Lin, H. Wang, and J.-j. Zhang, Thermality and excited state Rényi entropy in two-dimensional CFT, J. High Energy Phys. 11 (2016) 116.

[15] B. Chen, J.-B. Wu, and J.-j. Zhang, Short interval expansion of Rnyi entropy on torus, J. High Energy Phys. 08 (2016) 130.

[16] N. Lashkari, A. Dymarsky, and H. Liu, Eigenstate thermalization hypothesis in conformal field theory, J. Stat. Mech. (2018) 033101.
[17] P. Basu, D. Das, S. Datta, and S. Pal, Thermality of eigenstates in conformal field theories, Phys. Rev. E 96, 022149 (2017).

[18] T. Faulkner and H. Wang, Probing beyond ETH at large $c$, J. High Energy Phys. 06 (2018) 123.

[19] S. He, F.-L. Lin, and J.-j. Zhang, Dissimilarities of reduced density matrices and eigenstate thermalization hypothesis, J. High Energy Phys. 12 (2017) 073.

[20] S. He, F.-L. Lin, and J.-j. Zhang, Subsystem eigenstate thermalization hypothesis for entanglement entropy in CFT, J. High Energy Phys. 08 (2017) 126.

[21] W.-z. Guo, F.-L. Lin, and J. Zhang, Note on ETH of descendant states in 2D CFT, J. High Energy Phys. 01 (2019) 152.

[22] V. V. Bazhanov, S. L. Lukyanov, and A. B. Zamolodchikov, Integrable structure of conformal field theory, quantum KdV theory and thermodynamic Bethe ansatz, Commun. Math. Phys. 177, 381 (1996).

[23] V. V. Bazhanov, S. L. Lukyanov, and A. B. Zamolodchikov, Integrable structure of conformal field theory II. Q-operator and DDV equation, Commun. Math. Phys. 190, 247 (1997).

[24] V. V. Bazhanov, S. L. Lukyanov, and A. B. Zamolodchikov, Integrable structure of conformal field theory III. The YangBaxter relation, Commun. Math. Phys. 200, 297 (1999).

[25] P. Calabrese, F. H. L. Essler, and M. Fagotti, Quantum Quench in the Transverse-Field Ising Chain, Phys. Rev. Lett. 106, 227203 (2011).

[26] J. Cardy, Quantum quenches to a critical point in one dimension: Some further results, J. Stat. Mech. (2016) 023103.

[27] M. Brockmann, B. Wouters, D. Fioretto, J. De Nardis, R. Vlijm, and J.-S. Caux, Quench action approach for releasing the Néel state into the spin-1/2 XXZ chain, J. Stat. Mech. (2014) P12009.

[28] M. Mestyán, B. Pozsgay, G. Takács, and M. A. Werner, Quenching the $X X Z$ spin chain: Quench action approach versus generalized Gibbs ensemble, J. Stat. Mech. (2015) P04001.

[29] B. Wouters, J. De Nardis, M. Brockmann, D. Fioretto, M. Rigol, and J.-S. Caux, Quenching the Anisotropic Heisenberg Chain: Exact Solution and Generalized Gibbs Ensemble Predictions, Phys. Rev. Lett. 113, 117202 (2014).

[30] B. Pozsgay, M. Mestyán, M. A. Werner, M. Kormos, G. Zaránd, and G. Takács, Correlations after Quantum Quenches in the XXZ Spin Chain: Failure of the Generalized Gibbs Ensemble, Phys. Rev. Lett. 113, 117203 (2014).

[31] G. Goldstein and N. Andrei, Failure of the local generalized Gibbs ensemble for integrable models with bound states, Phys. Rev. A 90, 043625 (2014).

[32] E. Ilievski, M. Medenjak, and T. Prosen, Quasilocal Conserved Operators in the Isotropic Heisenberg Spin-1/2 Chain, Phys. Rev. Lett. 115, 120601 (2015).

[33] E. Ilievski, J. De Nardis, B. Wouters, J.-S. Caux, F. H. L. Essler, and T. Prosen, Complete Generalized Gibbs Ensembles in an Interacting Theory, Phys. Rev. Lett. 115, 157201 (2015)

[34] D. Fioretto and G. Mussardo, Quantum quenches in integrable field theories, New J. Phys. 12, 055015 (2010). 
[35] F. H. L. Essler, G. Mussardo, and M. Panfil, Generalized Gibbs ensembles for quantum field theories, Phys. Rev. A 91, 051602(R) (2015).

[36] S. Sotiriadis, Memory-preserving equilibration after a quantum quench in a one-dimensional critical model, Phys. Rev. A 94, 031605(R) (2016).

[37] B. Doyon, Thermalization and pseudolocality in extended quantum systems, Commun. Math. Phys. 351, 155 (2017).

[38] A. Bastianello and S. Sotiriadis, Quasi locality of the GGE in interacting-to-free quenches in relativistic field theories, J. Stat. Mech. (2017) 023105.

[39] T. Palmai and R. M. Konik, Quasilocal charges and the generalized Gibbs ensemble in the Lieb-Liniger model, Phys. Rev. E 98, 052126 (2018).

[40] E. Vernier and A.C. Cubero, Quasilocal charges and progress towards the complete GGE for field theories with nondiagonal scattering, J. Stat. Mech. (2017) 023101.

[41] A. Dymarsky and K. Pavlenko, Generalized gibbs ensemble of 2D CFTs at large central charge in the thermodynamic limit, J. High Energy Phys. 01 (2019) 098.

[42] J. de Boer and D. Engelhardt, Remarks on thermalization in 2D CFT, Phys. Rev. D 94, 126019 (2016).

[43] A. Pérez, D. Tempo, and R. Troncoso, Boundary conditions for general relativity on $\mathrm{AdS}_{3}$ and the KdV hierarchy, J. High Energy Phys. 06 (2016) 103.
[44] A. Maloney, G. Seng Ng, S. F. Ross, and I. Tsiares, Generalized Gibbs ensemble and the statistics of $\mathrm{KdV}$ charges in 2D CFT, J. High Energy Phys. 03 (2019) 075.

[45] See Supplemental Material at http://link.aps.org/ supplemental/10.1103/PhysRevLett.123.111602 for the proof that a typical KdV eigenstate satisfies Eq. (6).

[46] A. Dymarsky and K. Pavlenko, Exact generalized partition function of 2D CFTs at large central charge, J. High Energy Phys. 05 (2019) 077.

[47] N. Lashkari, A. Dymarsky, and H. Liu, Universality of quantum information in chaotic CFTs, J. High Energy Phys. 03 (2018) 070.

[48] S. Nandy, A. Sen, A. Das, and A. Dhar, Eigenstate Gibbs ensemble in integrable quantum systems, Phys. Rev. B 94, 245131 (2016).

[49] B. Pozsgay, E. Vernier, and M. A. Werner, On generalized Gibbs ensembles with an infinite set of conserved charges, J. Stat. Mech. (2017) 093103.

[50] P. W. Claeys, D. Van Neck, and S. De Baerdemacker, Inner products in integrable Richardson-Gaudin models, SciPost Phys. 3, 028 (2017).

[51] P. W. Claeys, Richardson-Gaudin models and broken integrability, Ph.D. thesis, Ghent University, 2018 [arXiv: 1809.04447]. 